\title{
Some New Reverses and Refinements of Inequalities for Relative Operator Entropy
}

\author{
S.S. DRAGOMIR ${ }^{1,2}$ \\ ${ }^{1}$ Mathematics, College of Engineering $\&$ Science, Victoria University \\ PO Box 14428, Melbourne City, MC 8001, Australia \\ sever.dragomir@vu.edu.au, http://rgmia.org/dragomir \\ ${ }^{2}$ School of Computer Science \& Applied Mathematics, University of the Witwatersrand \\ Private Bag 3, Johannesburg 2050, South Africa
}

Abstract: In this paper we obtain new inequalities for relative operator entropy $S(A \mid B)$ in the case of operators satisfying the condition $m A \leq B \leq M A$, with $0<m<M$.

Key words: Inequalities for Logarithm, Relative operator entropy, Operator entropy.

AMS Subject Class. (2000): 47A63, 47A30.

\section{INTRODUCTION}

Kamei and Fujii $[6,7]$ defined the relative operator entropy $S(A \mid B)$, for positive invertible operators $A$ and $B$, by

$$
S(A \mid B):=A^{\frac{1}{2}}\left(\ln \left(A^{-\frac{1}{2}} B A^{-\frac{1}{2}}\right)\right) A^{\frac{1}{2}}
$$

which is a relative version of the operator entropy considered by NakamuraUmegaki [12].

In general, we can define for positive operators $A, B$

$$
S(A \mid B):=s-\lim _{\varepsilon \rightarrow 0+} S\left(A+\varepsilon 1_{H} \mid B\right)
$$

if it exists, here $1_{H}$ is the identity operator.

For the entropy function $\eta(t)=-t \ln t$, the operator entropy has the following expression:

$$
\eta(A)=-A \ln A=S\left(A \mid 1_{H}\right) \geq 0
$$

for positive contraction $A$. This shows that the relative operator entropy (1.1) is a relative version of the operator entropy. 
Following [8, pp. 149-155], we recall some important properties of relative operator entropy for $A$ and $B$ positive invertible operators:

(i) We have the equalities

$$
\begin{aligned}
S(A \mid B) & =-A^{1 / 2}\left(\ln A^{1 / 2} B^{-1} A^{1 / 2}\right) A^{1 / 2} \\
& =B^{1 / 2} \eta\left(B^{-1 / 2} A B^{-1 / 2}\right) B^{1 / 2}
\end{aligned}
$$

(ii) We have the inequalities

$$
S(A \mid B) \leq A(\ln \|B\|-\ln A) \quad \text { and } \quad S(A \mid B) \leq B-A .
$$

(iii) For any $C, D$ positive invertible operators we have that

$$
S(A+B \mid C+D) \geq S(A \mid C)+S(B \mid D) .
$$

(iv) If $B \leq C$ then

$$
S(A \mid B) \leq S(A \mid C) .
$$

(v) If $B_{n} \downarrow B$ then

$$
S\left(A \mid B_{n}\right) \downarrow S(A \mid B) .
$$

(vi) For $\alpha>0$ we have

$$
S(\alpha A \mid \alpha B)=\alpha S(A \mid B) .
$$

(vii) For every operator $T$ we have

$$
T^{*} S(A \mid B) T \leq S\left(T^{*} A T \mid T^{*} B T\right) .
$$

The relative operator entropy is jointly concave, namely, for any positive invertible operators $A, B, C, D$ we have

$$
S(t A+(1-t) B \mid t C+(1-t) D) \geq t S(A \mid C)+(1-t) S(B \mid D)
$$

for any $t \in[0,1]$.

For other results on the relative operator entropy see $[1,4,9,10,11,13]$. Observe that, if we replace in (1.2) $B$ with $A$, then we get

$$
\begin{aligned}
S(B \mid A) & =A^{1 / 2} \eta\left(A^{-1 / 2} B A^{-1 / 2}\right) A^{1 / 2} \\
& =A^{1 / 2}\left(-A^{-1 / 2} B A^{-1 / 2} \ln \left(A^{-1 / 2} B A^{-1 / 2}\right)\right) A^{1 / 2},
\end{aligned}
$$


therefore we have

$$
A^{1 / 2}\left(A^{-1 / 2} B A^{-1 / 2} \ln \left(A^{-1 / 2} B A^{-1 / 2}\right)\right) A^{1 / 2}=-S(B \mid A)
$$

for positive invertible operators $A$ and $B$.

It is well know that, in general $S(A \mid B)$ is not equal to $S(B \mid A)$.

In [15], A. Uhlmann has shown that the relative operator entropy $S(A \mid B)$ can be represented as the strong limit

$$
S(A \mid B)=s-\lim _{t \rightarrow 0} \frac{A \sharp_{t} B-A}{t},
$$

where

$$
A \sharp_{\nu} B:=A^{1 / 2}\left(A^{-1 / 2} B A^{-1 / 2}\right)^{\nu} A^{1 / 2}, \quad \nu \in[0,1],
$$

is the weighted geometric mean of positive invertible operators $A$ and $B$. For $\nu=\frac{1}{2}$ we denote $A \sharp B$.

This definition of the weighted geometric mean can be extended for any real number $\nu$ with $\nu \neq 0$.

For $t>0$ and the positive invertible operators $A, B$ we define the Tsallis relative operator entropy (see also [3]) by

$$
T_{t}(A \mid B):=\frac{A \sharp_{t} B-A}{t} .
$$

The following result providing upper and lower bounds for relative operator entropy in terms of $T_{t}(\cdot \mid \cdot)$ has been obtained in [6] for $0<t \leq 1$. However, it hods for any $t>0$.

Theorem 1. Let $A, B$ be two positive invertible operators, then for any $t>0$ we have

$$
T_{t}(A \mid B)\left(A \sharp_{t} B\right)^{-1} A \leq S(A \mid B) \leq T_{t}(A \mid B) .
$$

In particular, we have for $t=1$ that

$$
\left(1_{H}-A B^{-1}\right) A \leq S(A \mid B) \leq B-A, \quad[6]
$$

and for $t=2$ that

$$
\frac{1}{2}\left(1_{H}-\left(A B^{-1}\right)^{2}\right) A \leq S(A \mid B) \leq \frac{1}{2}\left(B A^{-1} B-A\right) .
$$


The case $t=\frac{1}{2}$ is of interest as well. Since in this case we have

$$
T_{1 / 2}(A \mid B):=2(A \sharp B-A)
$$

and

$$
T_{1 / 2}(A \mid B)\left(A \sharp_{1 / 2} B\right)^{-1} A=2\left(1_{H}-A(A \sharp B)^{-1}\right) A,
$$

hence by (1.6) we get

$$
2\left(1_{H}-A(A \sharp B)^{-1}\right) A \leq S(A \mid B) \leq 2(A \sharp B-A) \leq B-A .
$$

Motivated by the above results, in this paper we obtain new inequalities for the relative operator entropy in the case of operators satisfying the condition $m A \leq B \leq M A$, with $0<m<M$.

\section{INEQUALITIES FOR LOG-FUNCTION}

We have:

Theorem 2. For any $a, b>0$ we have the inequalities

$$
\frac{1}{2 b \min \{a, b\}}(b-a)^{2} \geq \ln b-\ln a-\frac{b-a}{b} \geq \frac{1}{2 b \max \{a, b\}}(b-a)^{2}
$$

and

$$
\frac{1}{2 a \min \{a, b\}}(b-a)^{2} \geq \frac{b-a}{a}-\ln b+\ln a \geq \frac{1}{2 a \max \{a, b\}}(b-a)^{2} .
$$

Proof. We have

$$
\int_{a}^{b} \frac{b-t}{t} \mathrm{~d} t=b \int_{a}^{b} \frac{1}{t} \mathrm{~d} t-\int_{a}^{b} \mathrm{~d} t=b(\ln b-\ln a)-(b-a)
$$

giving that

$$
\ln b-\ln a-\frac{b-a}{b}=\frac{1}{b} \int_{a}^{b} \frac{b-t}{t} \mathrm{~d} t
$$

for any $a, b>0$.

Let $b>a>0$, then

$$
\frac{1}{a} \int_{a}^{b}(b-t) \mathrm{d} t \geq \int_{a}^{b} \frac{b-t}{t} \mathrm{~d} t \geq \frac{1}{b} \int_{a}^{b}(b-t) \mathrm{d} t
$$


giving that

$$
\frac{1}{2 a}(b-a)^{2} \geq \int_{a}^{b} \frac{b-t}{t} \mathrm{~d} t \geq \frac{1}{2 b}(b-a)^{2} .
$$

Let $a>b>0$, then

$$
\frac{1}{b} \int_{b}^{a}(t-b) \mathrm{d} t \geq \int_{a}^{b} \frac{b-t}{t} \mathrm{~d} t=\int_{b}^{a} \frac{t-b}{t} \mathrm{~d} t \geq \frac{1}{a} \int_{b}^{a}(t-b) \mathrm{d} t
$$

giving that

$$
\frac{1}{2 b}(b-a)^{2} \geq \int_{a}^{b} \frac{b-t}{t} \mathrm{~d} t \geq \frac{1}{2 a}(b-a)^{2} .
$$

Therefore, by (2.4) and (2.5) we get

$$
\frac{1}{2 \min \{a, b\}}(b-a)^{2} \geq \int_{a}^{b} \frac{b-t}{t} \mathrm{~d} t \geq \frac{1}{2 \max \{a, b\}}(b-a)^{2},
$$

for any $a, b>0$.

By utilising the equality (2.3) we get the desired result (2.1).

Corollary 1. For any $y>0$ we have

$$
\begin{gathered}
\frac{1}{2 y \min \{1, y\}}(y-1)^{2} \geq \ln y-\frac{y-1}{y} \geq \frac{1}{2 y \max \{1, y\}}(y-1)^{2}, \\
\frac{1}{2 \min \{1, y\}}(y-1)^{2} \geq y-1-\ln y \geq \frac{1}{2 \max \{1, y\}}(y-1)^{2} .
\end{gathered}
$$

Remark 1. Since for any $a, b>0$ we have $\max \{a, b\} \min \{a, b\}=a b$, then (2.1) and (2.2) can also be written as

$$
\begin{aligned}
\frac{1}{2 a} \max \{a, b\}\left(\frac{b-a}{b}\right)^{2} & \geq \ln b-\ln a-\frac{b-a}{b} \\
& \geq \frac{1}{2 a} \min \{a, b\}\left(\frac{b-a}{b}\right)^{2}
\end{aligned}
$$

and

$$
\begin{aligned}
\frac{1}{2 b} \max \{a, b\}\left(\frac{b-a}{a}\right)^{2} & \geq \frac{b-a}{a}-\ln b+\ln a \\
& \geq \frac{1}{2 b} \min \{a, b\}\left(\frac{b-a}{a}\right)^{2}
\end{aligned}
$$


for any $a, b>0$.

The inequalities can also be written as

$$
\frac{1}{2} \max \{1, y\}\left(\frac{y-1}{y}\right)^{2} \geq \ln y-\frac{y-1}{y} \geq \frac{1}{2} \min \{1, y\}\left(\frac{y-1}{y}\right)^{2}
$$

and

$$
\frac{1}{2 y} \max \{1, y\}(y-1)^{2} \geq y-1-\ln y \geq \frac{1}{2 y} \min \{1, y\}(y-1)^{2},
$$

for any $y>0$.

In the recent paper [2] we obtained the following inequalities that provide upper and lower bounds for the quantity $\ln b-\ln a-\frac{b-a}{b}$ :

$$
\frac{1}{2} \frac{(b-a)^{2}}{\min ^{2}\{a, b\}} \geq \frac{b-a}{a}-\ln b+\ln a \geq \frac{1}{2} \frac{(b-a)^{2}}{\max ^{2}\{a, b\}},
$$

where $a, b>0$ and

$$
\frac{(b-a)^{2}}{a b} \geq \frac{b-a}{a}-\ln b+\ln a
$$

for any $a, b>0$.

It is natural to ask, which of the upper bounds for the quantity

$$
\frac{b-a}{a}-\ln b+\ln a
$$

as provided by (2.2), (2.12) and (2.13) is better?

It has been shown in [2] that neither of the upper bounds in (2.12) and (2.13) is always best.

Consider now the difference

$$
\begin{aligned}
D_{1}(a, b) & :=\frac{1}{2 a \min \{a, b\}}(b-a)^{2}-\frac{1}{2} \frac{(b-a)^{2}}{\min ^{2}\{a, b\}} \\
& =\frac{1}{2} \frac{(b-a)^{2}}{a \min ^{2}\{a, b\}}(\min \{a, b\}-a) \leq 0,
\end{aligned}
$$

which shows that upper bound in (2.2) is always better than the upper bound in (2.12). 
Consider the difference

$$
\begin{aligned}
D_{2}(a, b): & =\frac{1}{2 a \min \{a, b\}}(b-a)^{2}-\frac{(b-a)^{2}}{a b} \\
& =\frac{1}{2 a b \min \{a, b\}}(b-a)^{2}(b-2 \min \{a, b\}),
\end{aligned}
$$

which can take both positive and negative values for $a, b>0$, showing that neither of the bounds (2.2) and (2.13) is always best.

Now, consider the difference

$$
\begin{aligned}
d(a, b): & =\frac{1}{2 a \max \{a, b\}}(b-a)^{2}-\frac{1}{2} \frac{(b-a)^{2}}{\max ^{2}\{a, b\}} \\
& =\frac{1}{2 a \max ^{2}\{a, b\}}(b-a)^{2}(\max \{a, b\}-a) \geq 0,
\end{aligned}
$$

which shows that lower bound in (2.2) is always better than the lower bound in $(2.12)$.

Corollary 2. If $y \in[k, K] \subset(0, \infty)$, then we have the local inequalities

$$
\begin{aligned}
\frac{1}{2 \min \{1, k\}} \frac{(y-1)^{2}}{y} & \geq \ln y-\frac{y-1}{y} \geq \frac{1}{2 \max \{1, K\}} \frac{(y-1)^{2}}{y}, \\
\frac{1}{2 \min \{1, k\}}(y-1)^{2} & \geq y-1-\ln y \geq \frac{1}{2 \max \{1, K\}}(y-1)^{2}, \\
\frac{1}{2} \max \{1, K\}\left(\frac{y-1}{y}\right)^{2} & \geq \ln y-\frac{y-1}{y} \geq \frac{1}{2} \min \{1, k\}\left(\frac{y-1}{y}\right)^{2}, \\
\frac{1}{2} \max \{1, K\} \frac{(y-1)^{2}}{y} & \geq y-1-\ln y \geq \frac{1}{2} \min \{1, k\} \frac{(y-1)^{2}}{y} .
\end{aligned}
$$

Proof. If $y \in[k, K] \subset(0, \infty)$, then by analyzing all possible locations of the interval $[k, K]$ and 1 we have

$$
\begin{gathered}
\min \{1, k\} \leq \min \{1, y\} \leq \min \{1, K\}, \\
\max \{1, k\} \leq \max \{1, y\} \leq \max \{1, K\} .
\end{gathered}
$$


By using the inequalities (2.6) and (2.7) we have

$$
\begin{aligned}
\frac{1}{2 y \min \{1, k\}}(y-1)^{2} & \geq \frac{1}{2 y \min \{1, y\}}(y-1)^{2} \\
& \geq \ln y-\frac{y-1}{y} \geq \frac{1}{2 y \max \{1, y\}}(y-1)^{2} \\
& \geq \frac{1}{2 y \max \{1, K\}}(y-1)^{2}
\end{aligned}
$$

and

$$
\begin{aligned}
\frac{1}{2 \min \{1, k\}}(y-1)^{2} & \geq \frac{1}{2 \min \{1, y\}}(y-1)^{2} \\
& \geq y-1-\ln y \geq \frac{1}{2 \max \{1, y\}}(y-1)^{2} \\
& \geq \frac{1}{2 \max \{1, K\}}(y-1)^{2}
\end{aligned}
$$

for any $y \in[k, K]$, that prove (2.14) and (2.15).

The inequalities (2.16) and (2.17) follows by (2.16) and (2.17).

If we consider the function $f(y)=\frac{(y-1)^{2}}{y}, y>0$, then we observe that

$$
f^{\prime}(y)=\frac{y^{2}-1}{y^{2}} \quad \text { and } \quad f^{\prime \prime}(y)=\frac{2}{y^{3}}
$$

which shows that $f$ is strictly decreasing on $(0,1)$, strictly increasing on $[1, \infty)$ and strictly convex for $y>0$. We also have $f\left(\frac{1}{y}\right)=f(y)$ for $y>0$.

By the properties of $f$ we then have that

$$
\begin{aligned}
& \max _{y \in[k, K]} \frac{(y-1)^{2}}{y}= \begin{cases}\frac{(k-1)^{2}}{k} & \text { if } K<1, \\
\max \left\{\frac{(k-1)^{2}}{k}, \frac{(K-1)^{2}}{K}\right\} & \text { if } k \leq 1 \leq K, \\
\frac{(K-1)^{2}}{K} & \text { if } 1<k,\end{cases} \\
& =: U(k, K)
\end{aligned}
$$


and

$$
\begin{aligned}
\min _{y \in[k, K]} \frac{(y-1)^{2}}{y} & = \begin{cases}\frac{(1-K)^{2}}{K} & \text { if } K<1, \\
0 & \text { if } k \leq 1 \leq K, \\
\frac{(k-1)^{2}}{k} & \text { if } 1<k,\end{cases} \\
& =: u(k, K) .
\end{aligned}
$$

We can provide now some global bounds as follows.

From (2.14) we then get for any $y \in[k, K]$ that

$$
\frac{1}{2 \min \{1, k\}} U(k, K) \geq \ln y-\frac{y-1}{y} \geq \frac{1}{2 \max \{1, K\}} u(k, K),
$$

while from (2.17) we get for any $y \in[k, K]$ that

$$
\frac{1}{2} \max \{1, K\} U(k, K) \geq y-1-\ln y \geq \frac{1}{2} \min \{1, k\} u(k, K) .
$$

Consider

$$
\begin{aligned}
Z(k, K): & =\max _{y \in[k, K]}(y-1)^{2} \\
& = \begin{cases}(1-k)^{2} & \text { if } K<1, \\
\max \left\{(1-k)^{2},(K-1)^{2}\right\} & \text { if } k \leq 1 \leq K, \\
(K-1)^{2} & \text { if } 1<k,\end{cases}
\end{aligned}
$$

and

$$
z(k, K):=\min _{y \in[k, K]}(y-1)^{2}= \begin{cases}(1-K)^{2} & \text { if } K<1 \\ 0 & \text { if } k \leq 1 \leq K \\ (k-1)^{2} & \text { if } 1<k\end{cases}
$$

By making use of (2.15) we get

$$
\frac{1}{2 \min \{1, k\}} Z(k, K) \geq y-1-\ln y \geq \frac{1}{2 \max \{1, K\}} z(k, K),
$$

for any $y \in[k, K]$. 
Consider the function $g(y)=\left(\frac{y-1}{y}\right)^{2}, y>0$, then we observe that

$$
g^{\prime}(y)=\frac{2(y-1)}{y^{2}} \quad \text { and } \quad g^{\prime \prime}(y)=\frac{2(3-2 y)}{y^{4}},
$$

which shows that $g$ is strictly decreasing on $(0,1)$, strictly increasing on $[1, \infty)$ strictly convex for $y \in(0,3 / 2)$ and strictly concave on $(3 / 2, \infty)$.

Consider

$$
\begin{aligned}
W(k, K): & =\max _{y \in[k, K]}\left(\frac{y-1}{y}\right)^{2} \\
& = \begin{cases}\left(\frac{1-k}{k}\right)^{2} & \text { if } K<1, \\
\max \left\{\left(\frac{1-k}{k}\right)^{2},\left(\frac{K-1}{K}\right)^{2}\right\} & \text { if } k \leq 1 \leq K, \\
\left(\frac{K-1}{K}\right)^{2} & \text { if } 1<k,\end{cases}
\end{aligned}
$$

and

$$
w(k, K):=\min _{y \in[k, K]}\left(\frac{y-1}{y}\right)^{2}= \begin{cases}\left(\frac{1-K}{K}\right)^{2} & \text { if } K<1 \\ 0 & \text { if } k \leq 1 \leq K \\ \left(\frac{k-1}{k}\right)^{2} & \text { if } 1<k .\end{cases}
$$

Then by $(2.16)$ we get

$$
\frac{1}{2} \max \{1, K\} W(k, K) \geq \ln y-\frac{y-1}{y} \geq \frac{1}{2} \min \{1, k\} w(k, K)
$$

for any $y \in[k, K]$.

\section{Operator inequalities}

We have the following:

Lemma 1. Let $x \in[k, K]$ and $t>0$, then we have

$$
\begin{aligned}
\frac{1}{2 \min \left\{1, k^{t}\right\}}\left(\frac{x^{t}-1}{t}-\frac{1-x^{-t}}{t}\right) & \\
& \geq \ln x-\frac{1-x^{-t}}{t} \\
& \geq \frac{1}{2 \max \left\{1, K^{t}\right\}}\left(\frac{x^{t}-1}{t}-\frac{1-x^{-t}}{t}\right) \geq 0
\end{aligned}
$$


and

$$
\begin{aligned}
\frac{1}{2} \max \left\{1, K^{t}\right\} t\left(\frac{1-x^{-t}}{t}\right)^{2} & \geq \ln x-\frac{1-x^{-t}}{t} \\
& \geq \frac{1}{2} \min \left\{1, k^{t}\right\} t\left(\frac{1-x^{-t}}{t}\right)^{2} \geq 0
\end{aligned}
$$

Proof. Let $y=x^{t} \in\left[k^{t}, K^{t}\right]$. By using the inequality (2.14) we have

$$
\begin{aligned}
\frac{1}{2 \min \left\{1, k^{t}\right\}}\left(x^{t}+x^{-t}-2\right) & \geq t \ln x-\frac{x^{t}-1}{x^{t}} \\
& \geq \frac{1}{2 \max \left\{1, K^{t}\right\}}\left(x^{t}+x^{-t}-2\right) \geq 0
\end{aligned}
$$

that is equivalent to (3.1).

From the inequality (2.16) we have for $y=x^{t}$

$$
\begin{aligned}
\frac{1}{2} \max \left\{1, K^{t}\right\}\left(1-2 x^{-t}+x^{-2 t}\right) & \geq t \ln x-\frac{x^{t}-1}{x^{t}} \\
& \geq \frac{1}{2} \min \left\{1, k^{t}\right\}\left(1-2 x^{-t}+x^{-2 t}\right) \geq 0
\end{aligned}
$$

that is equivalent to (3.2).

We have:

Theorem 3. Let $A, B$ be two positive invertible operators and the constants $M>m>0$ with the property that

$$
m A \leq B \leq M A \text {. }
$$

Then for any $t>0$ we have

$$
\begin{aligned}
\frac{1}{2 \min \left\{1, m^{t}\right\}} & T_{t}(A \mid B)\left(A^{-1}-\left(A \sharp_{t} B\right)^{-1}\right) A \\
& \geq S(A \mid B)-T_{t}(A \mid B)\left(A \sharp_{t} B\right)^{-1} A \\
& \geq \frac{1}{2 \max \left\{1, M^{t}\right\}} T_{t}(A \mid B)\left(A^{-1}-\left(A \sharp_{t} B\right)^{-1}\right) A \geq 0
\end{aligned}
$$


and

$$
\begin{aligned}
\frac{1}{2} \max \left\{1, M^{t}\right\} & t\left(T_{t}(A \mid B)\left(A \sharp_{t} B\right)^{-1}\right)^{2} A \\
& \geq S(A \mid B)-T_{t}(A \mid B)\left(A \sharp_{t} B\right)^{-1} A \\
& \geq \frac{1}{2} \min \left\{1, m^{t}\right\} t\left(T_{t}(A \mid B)\left(A \sharp_{t} B\right)^{-1}\right)^{2} A \geq 0 .
\end{aligned}
$$

Proof. Since $m A \leq B \leq M A$ and $A$ is invertible, then by multiplying both sides with $A^{-1 / 2}$ we get $m 1_{H} \leq A^{-1 / 2} B A^{-1 / 2} \leq M$. Denote $X=$ $A^{-1 / 2} B A^{-1 / 2}$ and by using the functional calculus for $X$ that has its spectrum contained in the interval $[m, M]$ and the inequality (3.1), we get

$$
\begin{aligned}
& \frac{1}{2 \min \left\{1, m^{t}\right\}}\left(\frac{\left(A^{-1 / 2} B A^{-1 / 2}\right)^{t}-1_{H}}{t}-\frac{1_{H}-\left(A^{-1 / 2} B A^{-1 / 2}\right)^{-t}}{t}\right) \\
& \geq \ln \left(A^{-1 / 2} B A^{-1 / 2}\right)-\frac{1_{H}-\left(A^{-1 / 2} B A^{-1 / 2}\right)^{-t}}{t} \\
& \geq \frac{1}{2 \max \left\{1, M^{t}\right\}}\left(\frac{\left(A^{-1 / 2} B A^{-1 / 2}\right)^{t}-1_{H}}{t}-\frac{1_{H}-\left(A^{-1 / 2} B A^{-1 / 2}\right)^{-t}}{t}\right) \\
& \geq 0
\end{aligned}
$$

for any $t>0$.

Now, if we multiply both sides of (3.6) by $A^{1 / 2}$, then we get

$$
\begin{gathered}
\frac{1}{2 \min \left\{1, m^{t}\right\}} A^{1 / 2}\left(\frac{\left(A^{-1 / 2} B A^{-1 / 2}\right)^{t}-1_{H}}{t}-\frac{1_{H}-\left(A^{-1 / 2} B A^{-1 / 2}\right)^{-t}}{t}\right) A^{1 / 2} \\
\geq A^{1 / 2}\left(\ln \left(A^{-1 / 2} B A^{-1 / 2}\right)\right) A^{1 / 2}-A^{1 / 2} \frac{1_{H}-\left(A^{-1 / 2} B A^{-1 / 2}\right)^{-t}}{t} A^{1 / 2} \\
\geq \frac{1}{2 \max \left\{1, M^{t}\right\}} A^{1 / 2}\left(\frac{\left(A^{-1 / 2} B A^{-1 / 2}\right)^{t}-1_{H}}{t}\right. \\
\left.-\frac{1_{H}-\left(A^{-1 / 2} B A^{-1 / 2}\right)-t}{t}\right) A^{1 / 2} \geq 0
\end{gathered}
$$

for any $t>0$.

Observe that

$$
A^{1 / 2} \ln \left(A^{-1 / 2} B A^{-1 / 2}\right) A^{1 / 2}=S(A \mid B),
$$




$$
\begin{aligned}
& A^{1 / 2} \frac{\left(A^{-1 / 2} B A^{-1 / 2}\right)^{t}-1}{t} A^{1 / 2}=\frac{A_{t} B-A}{t}=T_{t}(A \mid B), \\
A^{1 / 2} \frac{1_{H}-\left(A^{-1 / 2} B A^{-1 / 2}\right)^{-t}}{t} A^{1 / 2} & A^{1 / 2} \frac{\left(A^{-1 / 2} B A^{-1 / 2}\right)^{t}\left(A^{-1 / 2} B A^{-1 / 2}\right)^{-t}-\left(A^{-1 / 2} B A^{-1 / 2}\right)^{-t}}{t} A^{1 / 2} \\
= & A^{1 / 2} \frac{\left(A^{-1 / 2} B A^{-1 / 2}\right)^{t}-1_{H}}{t}\left(A^{-1 / 2} B A^{-1 / 2}\right)^{-t} A^{1 / 2} \\
= & A^{1 / 2} \frac{\left(A^{-1 / 2} B A^{-1 / 2}\right)^{t}-1_{H}}{t} A^{1 / 2} A^{-1 / 2}\left(A^{-1 / 2} B A^{-1 / 2}\right)^{-t} A^{-1 / 2} A \\
= & T_{t}(A \mid B)\left(A \sharp_{t} B\right)^{-1} A
\end{aligned}
$$

and then by (3.7) we get

$$
\begin{aligned}
\frac{1}{2 \min \left\{1, m^{t}\right\}} & T_{t}(A \mid B)\left(1_{H}-\left(A \sharp_{t} B\right)^{-1} A\right) \\
& \geq S(A \mid B)-T_{t}(A \mid B)\left(A \sharp_{t} B\right)^{-1} A \\
& \geq \frac{1}{2 \max \left\{1, M^{t}\right\}} T_{t}(A \mid B)\left(1_{H}-\left(A \sharp_{t} B\right)^{-1} A\right) \geq 0
\end{aligned}
$$

that is equivalent to (3.4).

From the inequality (3.2) we also have

$$
\begin{aligned}
\frac{1}{2} \max \left\{1, M^{t}\right\} & \left(\frac{1_{H}-\left(A^{-1 / 2} B A^{-1 / 2}\right)^{-t}}{t}\right)^{2} \\
& \geq \ln \left(A^{-1 / 2} B A^{-1 / 2}\right)-\frac{1_{H}-\left(A^{-1 / 2} B A^{-1 / 2}\right)^{-t}}{t} \\
& \geq \frac{1}{2} \min \left\{1, m^{t}\right\} t\left(\frac{1_{H}-\left(A^{-1 / 2} B A^{-1 / 2}\right)^{-t}}{t}\right)^{2} \geq 0 .
\end{aligned}
$$


Now, if we multiply both sides of (3.9) by $A^{1 / 2}$, then we get

$$
\begin{aligned}
\frac{1}{2} \max & \left\{1, M^{t}\right\} t A^{1 / 2}\left(\frac{1_{H}-\left(A^{-1 / 2} B A^{-1 / 2}\right)^{-t}}{t}\right)^{2} A^{1 / 2} \\
& \geq A^{1 / 2}\left(\ln \left(A^{-1 / 2} B A^{-1 / 2}\right)\right) A^{1 / 2}-A^{1 / 2} \frac{1_{H}-\left(A^{-1 / 2} B A^{-1 / 2}\right)^{-t}}{t} A^{1 / 2} \\
& \geq \frac{1}{2} \min \left\{1, m^{t}\right\} t A^{1 / 2}\left(\frac{1_{H}-\left(A^{-1 / 2} B A^{-1 / 2}\right)^{-t}}{t}\right)^{2} A^{1 / 2} \geq 0
\end{aligned}
$$

From (3.8) we have, by multiplying both sides by $A^{-1 / 2}$, that

$$
\frac{1_{H}-\left(A^{-1 / 2} B A^{-1 / 2}\right)^{-t}}{t}=A^{-1 / 2} T_{t}(A \mid B)\left(A \sharp_{t} B\right)^{-1} A^{1 / 2} .
$$

Then

$$
\begin{aligned}
A^{1 / 2} & \left(\frac{1_{H}-\left(A^{-1 / 2} B A^{-1 / 2}\right)^{-t}}{t}\right)^{2} A^{1 / 2} \\
& =A^{1 / 2}\left(A^{-1 / 2} T_{t}(A \mid B)\left(A \sharp_{t} B\right)^{-1} A^{1 / 2}\right)^{2} A^{1 / 2} \\
& =A^{1 / 2} A^{-1 / 2} T_{t}(A \mid B)\left(A \sharp_{t} B\right)^{-1} A^{1 / 2} A^{-1 / 2} T_{t}(A \mid B)\left(A_{t} B\right)^{-1} A^{1 / 2} A^{1 / 2} \\
& =T_{t}(A \mid B)\left(A \sharp_{t} B\right)^{-1} T_{t}(A \mid B)\left(A \sharp_{t} B\right)^{-1} A \\
& =\left(T_{t}(A \mid B)\left(A \sharp_{t} B\right)^{-1}\right)^{2} A,
\end{aligned}
$$

which together with (3.10) produces the desired result (3.5).

There are some particular inequalities of interest as follows.

For $t=1$ we get from (3.4) and (3.5) that

$$
\begin{aligned}
\frac{1}{2 \min \{1, m\}} & (B-A)\left(A^{-1}-B^{-1}\right) A \\
& \geq S(A \mid B)-\left(1_{H}-A B^{-1}\right) A \\
& \geq \frac{1}{2 \max \{1, M\}}(B-A)\left(A^{-1}-B^{-1}\right) A \geq 0
\end{aligned}
$$


and

$$
\begin{aligned}
\frac{1}{2} \max \{1, M\} & \left(1_{H}-A B^{-1}\right)^{2} A \\
& \geq S(A \mid B)-\left(1_{H}-A B^{-1}\right) A \\
& \geq \frac{1}{2} \min \{1, m\}\left(1_{H}-A B^{-1}\right)^{2} A \geq 0 .
\end{aligned}
$$

For $t=1 / 2$ we get from (3.4) and (3.5) that

$$
\begin{aligned}
& \frac{1}{\min \{1, \sqrt{m}\}}(A \sharp B-A)\left(A^{-1}-(A \sharp B)^{-1}\right) A \\
& \geq S(A \mid B)-2\left(1_{H}-A(A \sharp B)^{-1}\right) A \\
& \geq \frac{1}{\max \{1, \sqrt{M}\}}(A \sharp B-A)\left(A^{-1}-(A \sharp B)^{-1}\right) A \geq 0
\end{aligned}
$$

and

$$
\begin{aligned}
\max \{1, \sqrt{M}\} & \left(1_{H}-A(A \sharp B)^{-1}\right)^{2} A \\
& \geq S(A \mid B)-2\left(1_{H}-A(A \sharp B)^{-1}\right) A \\
& \geq \min \{1, \sqrt{m}\}\left(1_{H}-A(A \sharp B)^{-1}\right)^{2} A \geq 0 .
\end{aligned}
$$

For $t=2$ we get from (3.4) and (3.5) that

$$
\begin{aligned}
& \frac{1}{4 \min \left\{1, m^{2}\right\}}\left(B A^{-1} B-A\right)\left(A^{-1}-B^{-1} A B^{-1}\right) A \\
& \quad \geq S(A \mid B)-\frac{1}{2}\left(1_{H}-\left(A B^{-1}\right)^{2}\right) A \\
& \quad \geq \frac{1}{4 \max \left\{1, M^{2}\right\}}\left(B A^{-1} B-A\right)\left(A^{-1}-B^{-1} A B^{-1}\right) A \geq 0
\end{aligned}
$$

and

$$
\begin{aligned}
\frac{1}{4} \max \left\{1, M^{2}\right\} & \left(1_{H}-\left(A B^{-1}\right)^{2}\right)^{2} A \\
& \geq S(A \mid B)-\frac{1}{2}\left(1_{H}-\left(A B^{-1}\right)^{2}\right) A \\
& \geq \frac{1}{4} \min \left\{1, m^{2}\right\}\left(1_{H}-\left(A B^{-1}\right)^{2}\right)^{2} A \geq 0 .
\end{aligned}
$$

We have the following: 
Lemma 2. Let $x \in[m, M]$ and $t>0$, then we have

$$
\begin{aligned}
\frac{1}{2 \min \left\{1, m^{t}\right\}} t\left(\frac{x^{t}-1}{t}\right)^{2} & \geq \frac{x^{t}-1}{t}-\ln x \\
& \geq \frac{1}{2 \max \left\{1, M^{t}\right\}} t\left(\frac{x^{t}-1}{t}\right)^{2}
\end{aligned}
$$

and

$$
\begin{aligned}
\frac{1}{2} \max \left\{1, M^{t}\right\}\left(\frac{x^{t}-1}{t}-\frac{1-x^{-t}}{t}\right) & \geq \frac{x^{t}-1}{t}-\ln x \\
& \geq \frac{1}{2} \min \left\{1, m^{t}\right\}\left(\frac{x^{t}-1}{t}-\frac{1-x^{-t}}{t}\right) .
\end{aligned}
$$

Proof. Let $y=x^{t} \in\left[m^{t}, M^{t}\right]$. By using the inequality (2.15) we have (3.17) and by (2.17) we have (3.18).

We also have:

ThEOREM 4. Let $A, B$ be two positive invertible operators and the constants $M>m>0$ with the property (3.3). Then for any $t>0$ we have

$$
\begin{aligned}
\frac{1}{2 \min \left\{1, m^{t}\right\}} t & T_{t}(A \mid B) A^{-1} T_{t}(A \mid B) \\
& \geq T_{t}(A \mid B)-S(A \mid B) \\
& \geq \frac{1}{2 \max \left\{1, M^{t}\right\}} t T_{t}(A \mid B) A^{-1} T_{t}(A \mid B) \geq 0
\end{aligned}
$$

and

$$
\begin{aligned}
\frac{1}{2} \max \left\{1, M^{t}\right\} & T_{t}(A \mid B)\left(1_{H}-\left(A \sharp_{t} B\right)^{-1} A\right) \\
& \geq T_{t}(A \mid B)-S(A \mid B) \\
& \geq \frac{1}{2} \min \left\{1, m^{t}\right\} T_{t}(A \mid B)\left(1_{H}-\left(A \sharp_{t} B\right)^{-1} A\right) \geq 0 .
\end{aligned}
$$

Proof. If we use the inequality (3.17) for the selfadjoint operator $X=$ $A^{-1 / 2} B A^{-1 / 2}$ that has its spectrum contained in the interval $[m, M]$, then we 
get

$$
\begin{aligned}
\frac{1}{2 \min \left\{1, m^{t}\right\}} & t\left(\frac{\left(A^{-1 / 2} B A^{-1 / 2}\right)^{t}-1}{t}\right)^{2} \\
& \geq \frac{\left(A^{-1 / 2} B A^{-1 / 2}\right)^{t}-1}{t}-\ln \left(A^{-1 / 2} B A^{-1 / 2}\right) \\
& \geq \frac{1}{2 \max \left\{1, M^{t}\right\}} t\left(\frac{\left(A^{-1 / 2} B A^{-1 / 2}\right)^{t}-1}{t}\right)^{2} \geq 0
\end{aligned}
$$

for any $t>0$.

If we multiply both sides of this inequality by $A^{1 / 2}$ we get

$$
\begin{aligned}
& \frac{1}{2 \min \left\{1, m^{t}\right\}} t A^{1 / 2}\left(\frac{\left(A^{-1 / 2} B A^{-1 / 2}\right)^{t}-1}{t}\right)^{2} A^{1 / 2} \\
& \geq A^{1 / 2} \frac{\left(A^{-1 / 2} B A^{-1 / 2}\right)^{t}-1}{t} A^{1 / 2}-A^{1 / 2}\left(\ln \left(A^{-1 / 2} B A^{-1 / 2}\right)\right) A^{1 / 2} \\
& \geq \frac{1}{2 \max \left\{1, M^{t}\right\}} t A^{1 / 2}\left(\frac{\left(A^{-1 / 2} B A^{-1 / 2}\right)^{t}-1}{t}\right)^{2} A^{1 / 2} \geq 0
\end{aligned}
$$

for any $t>0$.

Since

$$
A^{1 / 2} \frac{\left(A^{-1 / 2} B A^{-1 / 2}\right)^{t}-1}{t} A^{1 / 2}=T_{t}(A \mid B),
$$

then

$$
\frac{\left(A^{-1 / 2} B A^{-1 / 2}\right)^{t}-1}{t}=A^{-1 / 2} T_{t}(A \mid B) A^{-1 / 2}
$$

and

$$
\begin{aligned}
& A^{1 / 2}\left(\frac{\left(A^{-1 / 2} B A^{-1 / 2}\right)^{t}-1}{t}\right)^{2} A^{1 / 2} \\
& \quad=A^{1 / 2} A^{-1 / 2} T_{t}(A \mid B) A^{-1 / 2} A^{-1 / 2} T_{t}(A \mid B) A^{-1 / 2} A^{1 / 2} \\
& \quad=T_{t}(A \mid B) A^{-1} T_{t}(A \mid B)
\end{aligned}
$$

for any $t>0$. 
By making use of (3.21) we then get (3.19).

By using inequality (3.18) we have

$$
\begin{aligned}
\frac{1}{2} \max & \left\{1, M^{t}\right\}\left(\frac{\left(A^{-1 / 2} B A^{-1 / 2}\right)^{t}-1}{t}-\frac{1-\left(A^{-1 / 2} B A^{-1 / 2}\right)^{-t}}{t}\right) \\
& \geq \frac{\left(A^{-1 / 2} B A^{-1 / 2}\right)^{t}-1}{t}-\ln \left(A^{-1 / 2} B A^{-1 / 2}\right) \\
& \geq \frac{1}{2} \min \left\{1, m^{t}\right\}\left(\frac{\left(A^{-1 / 2} B A^{-1 / 2}\right)^{t}-1}{t}-\frac{1-\left(A^{-1 / 2} B A^{-1 / 2}\right)^{-t}}{t}\right) \geq 0,
\end{aligned}
$$

for any $t>0$.

If we multiply both sides of this inequality by $A^{1 / 2}$ we get

$$
\begin{aligned}
& \frac{1}{2} \max \left\{1, M^{t}\right\} A^{1 / 2}\left(\frac{\left(A^{-1 / 2} B A^{-1 / 2}\right)^{t}-1}{t}-\frac{1-\left(A^{-1 / 2} B A^{-1 / 2}\right)^{-t}}{t}\right) A^{1 / 2} \\
& \quad \geq A^{1 / 2} \frac{\left(A^{-1 / 2} B A^{-1 / 2}\right)^{t}-1}{t} A^{1 / 2}-A^{1 / 2}\left(\ln \left(A^{-1 / 2} B A^{-1 / 2}\right)\right) A^{1 / 2} \\
& \quad \geq \frac{1}{2} \min \left\{1, m^{t}\right\} A^{1 / 2}\left(\frac{\left(A^{-1 / 2} B A^{-1 / 2}\right)^{t}-1}{t}-\frac{1-\left(A^{-1 / 2} B A^{-1 / 2}\right)^{-t}}{t}\right) A^{1 / 2} \\
& \quad \geq 0
\end{aligned}
$$

for any $t>0$, and the inequality (3.20) is obtained.

For $t=1$ we get from (3.19) and (3.20) that

$$
\begin{aligned}
\frac{1}{2 \min \{1, m\}} & (B-A) A^{-1}(B-A) \\
& \geq B-A-S(A \mid B) \\
& \geq \frac{1}{2 \max \{1, M\}}(B-A) A^{-1}(B-A) \geq 0
\end{aligned}
$$

and

$$
\begin{aligned}
\frac{1}{2} \max \{1, M\} & (B-A)\left(1_{H}-B^{-1} A\right) \\
& \geq B-A-S(A \mid B) \\
& \geq \frac{1}{2} \min \{1, m\}(B-A)\left(1_{H}-B^{-1} A\right) \geq 0
\end{aligned}
$$


For $t=1 / 2$ we get from (3.19) and (3.20) that

$$
\begin{aligned}
\frac{1}{\min \{1, \sqrt{m}\}} & (A \sharp B-A) A^{-1}(A \sharp B-A) \\
& \geq 2(A \sharp B-A)-S(A \mid B) \\
& \geq \frac{1}{\max \{1, \sqrt{M}\}}(A \sharp B-A) A^{-1}(A \sharp B-A) \geq 0
\end{aligned}
$$

and

$$
\begin{aligned}
\max \{1, \sqrt{M}\} & (A \sharp B-A)\left(1_{H}-(A \sharp B)^{-1} A\right) \\
& \geq 2(A \sharp B-A)-S(A \mid B) \\
& \geq \min \{1, \sqrt{m}\}(A \sharp B-A)\left(1_{H}-(A \sharp B)^{-1} A\right) \geq 0 .
\end{aligned}
$$

For $t=2$ we get from (3.19) and (3.20) that

$$
\begin{aligned}
\frac{1}{4 \min \left\{1, m^{2}\right\}} & \left(B A^{-1} B-A\right) A^{-1}\left(B A^{-1} B-A\right) \\
& \geq \frac{1}{2}\left(B A^{-1} B-A\right)-S(A \mid B) \\
& \geq \frac{1}{4 \max \left\{1, M^{2}\right\}}\left(B A^{-1} B-A\right) A^{-1}\left(B A^{-1} B-A\right) \geq 0
\end{aligned}
$$

and

$$
\begin{aligned}
\frac{1}{4} \max \left\{1, M^{2}\right\} & \left(B A^{-1} B-A\right)\left(1_{H}-\left(B^{-1} A\right)^{2}\right) \\
& \geq \frac{1}{2}\left(B A^{-1} B-A\right)-S(A \mid B) \\
& \geq \frac{1}{4} \min \left\{1, m^{2}\right\}\left(B A^{-1} B-A\right)\left(1_{H}-\left(B^{-1} A\right)^{2}\right) \geq 0 .
\end{aligned}
$$

\section{Some Global BOUndS}

For $[m, M] \subset(0, \infty)$ and $t>0$ and by the use of (2.18) we define

$$
\begin{aligned}
U_{t}(m, M): & =U\left(m^{t}, M^{t}\right) \\
& = \begin{cases}\frac{\left(m^{t}-1\right)^{2}}{m^{t}} & \text { if } M<1, \\
\max \left\{\frac{\left(m^{t}-1\right)^{2}}{m^{t}}, \frac{\left(M^{t}-1\right)^{2}}{M^{t}}\right\} & \text { if } m \leq 1 \leq M, \\
\frac{\left(M^{t}-1\right)^{2}}{M^{t}} & \text { if } 1<m,\end{cases}
\end{aligned}
$$


and by (2.19)

$$
u_{t}(m, M):=u\left(m^{t}, M^{t}\right)= \begin{cases}\frac{\left(1-M^{t}\right)^{2}}{M^{t}} & \text { if } M<1, \\ 0 & \text { if } m \leq 1 \leq M, \\ \frac{\left(m^{t}-1\right)^{2}}{m^{t}} & \text { if } 1<m .\end{cases}
$$

By (2.20) and (2.21) we have for $y=x^{t} \in\left[m^{t}, M^{t}\right]$ and $t>0$ that

$$
\begin{aligned}
\frac{1}{2 t \min \left\{1, m^{t}\right\}} U_{t}(m, M) & \geq \ln x-\frac{1-x^{-t}}{t} \\
& \geq \frac{1}{2 t \max \left\{1, M^{t}\right\}} u_{t}(m, M)
\end{aligned}
$$

and

$$
\begin{aligned}
\frac{1}{2 t} \max \left\{1, M^{t}\right\} U_{t}(m, M) & \geq \frac{x^{t}-1}{t}-\ln x \\
& \geq \frac{1}{2 t} \min \left\{1, m^{t}\right\} u_{t}(m, M),
\end{aligned}
$$

where $x \in[m, M]$ and $t>0$.

Using (2.22) and (2.23) we define

$$
\begin{aligned}
Z_{t}(m, M):= & Z\left(m^{t}, M^{t}\right) \\
& = \begin{cases}\left(1-m^{t}\right)^{2} & \text { if } M<1, \\
\max \left\{\left(1-m^{t}\right)^{2},\left(M^{t}-1\right)^{2}\right\} & \text { if } m \leq 1 \leq M, \\
\left(M^{t}-1\right)^{2} & \text { if } 1<m,\end{cases}
\end{aligned}
$$

and

$$
z_{t}(m, M):=z\left(m^{t}, M^{t}\right)= \begin{cases}\left(1-M^{t}\right)^{2} & \text { if } M<1, \\ 0 & \text { if } m \leq 1 \leq M, \\ \left(m^{t}-1\right)^{2} & \text { if } 1<m .\end{cases}
$$

By (2.24) we have for $y=x^{t} \in\left[m^{t}, M^{t}\right]$ and $t>0$ that

$$
\begin{aligned}
\frac{1}{2 t \min \left\{1, m^{t}\right\}} Z_{t}(m, M) & \geq \frac{x^{t}-1}{t}-\ln x \\
& \geq \frac{1}{2 t \max \left\{1, M^{t}\right\}} z_{t}(m, M),
\end{aligned}
$$


where $x \in[m, M]$ and $t>0$.

Utilising (2.25) and (2.26) we can define

$$
\begin{aligned}
W_{t}(m, M): & =W\left(m^{t}, M^{t}\right) \\
& = \begin{cases}\left(\frac{1-m^{t}}{m^{t}}\right)^{2} & \text { if } M<1, \\
\max \left\{\left(\frac{1-m^{t}}{m^{t}}\right)^{2},\left(\frac{M^{t}-1}{M^{t}}\right)^{2}\right\} & \text { if } m \leq 1 \leq M, \\
\left(\frac{M^{t}-1}{M^{t}}\right)^{2} & \text { if } 1<m,\end{cases}
\end{aligned}
$$

and

$$
w_{t}(m, M):=W\left(m^{t}, M^{t}\right)= \begin{cases}\left(\frac{1-M^{t}}{M^{t}}\right)^{2} & \text { if } M<1, \\ 0 & \text { if } m \leq 1 \leq M, \\ \left(\frac{m^{t}-1}{m^{t}}\right)^{2} & \text { if } 1<m .\end{cases}
$$

By (2.24) we have for $y=x^{t} \in\left[m^{t}, M^{t}\right]$ and $t>0$ that

$$
\begin{aligned}
\frac{1}{2 t} \max \left\{1, M^{t}\right\} W_{t}(m, M) & \geq \ln x-\frac{1-x^{-t}}{t} \\
& \geq \frac{1}{2 t} \min \left\{1, m^{t}\right\} w_{t}(m, M),
\end{aligned}
$$

where $x \in[m, M]$ and $t>0$.

Theorem 5. Let $A, B$ be two positive invertible operators and the constants $M>m>0$ with the property (3.3). Then for any $t>0$ we have

$$
\begin{aligned}
\frac{1}{2 t \min \left\{1, m^{t}\right\}} U_{t}(m, M) A & \geq S(A \mid B)-T_{t}(A \mid B)\left(A \sharp_{t} B\right)^{-1} A \\
& \geq \frac{1}{2 t \max \left\{1, M^{t}\right\}} u_{t}(m, M) A, \\
\frac{1}{2 t} \max \left\{1, M^{t}\right\} W_{t}(m, M) A & \geq S(A \mid B)-T_{t}(A \mid B)\left(A \sharp_{t} B\right)^{-1} A \\
& \geq \frac{1}{2 t} \min \left\{1, m^{t}\right\} w_{t}(m, M) A, \\
\frac{1}{2 t \min \left\{1, m^{t}\right\}} Z_{t}(m, M) A & \geq T_{t}(A \mid B)-S(A \mid B) \\
& \geq \frac{1}{2 t \max \left\{1, M^{t}\right\}} z_{t}(m, M) A
\end{aligned}
$$


and

$$
\begin{aligned}
\frac{1}{2 t} \max \left\{1, M^{t}\right\} U_{t}(m, M) A & \geq T_{t}(A \mid B)-S(A \mid B) \\
& \geq \frac{1}{2 t} \min \left\{1, m^{t}\right\} u_{t}(m, M) A
\end{aligned}
$$

The proof follows by the inequalities (4.4), (4.5), (4.7) and (4.10) in a similar way as the one from the proof of Theorem 3 and we omit the details.

For $t=1, t=1 / 2$ and $t=2$ one can obtain some particular inequalities of interest, however the details are not provided here.

\section{REFERENCES}

[1] S.S. Dragomir, Some inequalities for relative operator entropy; preprint RGMIA Res. Rep. Coll. 18 (2015), Art. 145. [http://rgmia.org/papers/v18/v18a145.pdf] .

[2] S.S. Dragomir, Reverses and refinements of several inequalities for relative operator entropy; preprint RGMIA Res. Rep. Coll. 19 (2015). [http://rgmia.org/papers/v19/] .

[3] S. Furuichi, K. Yanagi, K. Kuriyama, Fundamental properties of Tsallis relative entropy, J. Math. Phys. 45 (2004) 4868-4877.

[4] S. Furuichi, Precise estimates of bounds on relative operator entropies, Math. Inequal. Appl. 18 (2015), 869-877.

[5] S. Furuichi, N. Minculete, Alternative reverse inequalities for Young's inequality, J. Math. Inequal. 5 (2011), Number 4, 595-600.

[6] J.I. FuJII, E. KAMEI, Uhlmann's interpolational method for operator means, Math. Japon. 34(4) (1989), 541-547.

[7] J.I. FuJII, E. KAMEI, Relative operator entropy in noncommutative information theory, Math. Japon. 34 (3) (1989), 341-348.

[8] T. Furuta, J. Mićić Hot, J. PeČArić, Y. Seo, "Mond-Pečarić Method in Operator Inequalities. Inequalities for Bounded Selfadjoint Operators on a Hilbert Space", Monographs in Inequalities 1, ELEMENT, Zagreb, 2005.

[9] I.H. KIM, Operator extension of strong subadditivity of entropy, J. Math. Phys. 53 (2012), 122204, 3 pp.

[10] P. Kluza, M. NiezGodA, Inequalities for relative operator entropies, Electron. J. Linear Algebra 27 (2014), 851-864.

[11] M.S. Moslehian, F. Mirzapour, A. Morassaei, Operator entropy inequalities, Colloq. Math. 130 (2013), 159-168.

[12] M. Nakamura, H. Umegaki, A note on the entropy for operator algebras, Proc. Japan Acad. 37 (1961) 149-154. 
[13] I. NikOUFAR, On operator inequalities of some relative operator entropies, Adv. Math. 259 (2014), 376-383.

[14] W. Specht, Zur Theorie der elementaren Mittel, Math. Z. 74 (1960), 91-98.

[15] A. Uhlmann, Relative entropy and the Wigner-Yanase-Dyson-Lieb concavity in an interpolation theory, Comm. Math. Phys. 54(1) (1977), 21-32. 
